effectiveness of treatments. The use of ionising radiation in radiology, however, is not without risks for health professionals directly involved in radiation work.

Methods The study was cross-sectional and prospective, including medical imaging technicians and engineers selected radiology departments, regularly hired and assigned to radiation work. Data were reported on a self-administered questionnaire.

Results Five hospitals were selected with 59 participants, including 6 (9\%) women, $54(92 \%)$ senior technicians and 5 $(8 \%)$ engineers. The cumulative age group of 30-50 years constituted $95 \%$ of the participants; 16 (27\%) participants had been exposed for 5-10 years, 2 (4\%) had been exposed for more than 20 years; The risk of cancer (96.6\%), radiodermatitis (31\%) and infertility (71.4\%) were recognised by the participants. The main PPEs identified by the participants as radio-protectors were the lead apron (96.6\%), the leaded glove $(68.6 \%)$, the shells $(31 \%)$, the anti-RX goggles $(57.8 \%)$; $33.2 \%$ of the participants wore them regularly, $60.6 \%$ were irregular, $6.2 \%$ did not wear them. PPE was available for $37.8 \%$ of the presentations. The interest of the dosimeter was known to $94.4 \%$ of the participants. Apparatus was revised in $19 \%$ of cases; $91 \%$ of participants received IR training and were qualified to work under radiation. Pictograms existed in $40 \%$ of hospitals, light signals in $80 \% ; 58.2 \%$ of the presentations knew their meaning.

Discussion The low availability and irregular wearing of PPE, and the ignorance of hazard indicators are more likely to expose them to IR.

Conclusion Strengthen protection measures through the availability of PPE and training

\section{OCCUPATIONAL SKIN CANCER IN OUTDOOR WORKERS IN ITALY: EXPECTED NUMBER VS CASES RECOGNISED BY THE ITALIAN NATIONAL COMPENSATION AUTHORITY (INAIL)}

${ }^{1}$ Alberto Modenese*, 'Leena Korpinen, ${ }^{1}$ Fabriziomaria Gobba. ${ }^{1}$ Department of Biomedical, Metabolic and Neural Sciences, University of Modena and Reggio Emilia, Modena, Italy; ${ }^{2}$ Clinical Physiology and Neurophysiology Unit, North Karelia Central Hospital and Honkalampi, Joensuu, Finland

\subsection{6/oemed-2018-ICOHabstracts.1229}

Introduction Solar Ultraviolet Radiation (UV) is one of the main risk factors for Non Melanoma Skin Cancer (NMSC) and Malignant Melanoma (MM). In Italy, only considering agriculture, fishery and construction sectors, the approximate number of workers exposed to solar UV (Outdoor workers OW-) is 2 million (1.6 million males, 400000 females).

Our aim is to compare the number of skin cancers (SCs) expected in OW to the number recognised by the Italian National Compensation Authority (INAIL).

Methods We collected data of Italian National Cancer Registres and the INAIL database of occupational diseases, including cancer, respectively available on the websites www. registri-tumori.it and www.bancadaticsa.inail.it

Results In Italy the yearly incidence of $\mathrm{MM}$ is 14,2 per 100000 in males and 13 per 100000 in females, that of NMSC 119.4 per 100000 in males and 90.7 per 100000 in females.
Applying these incidence rates to the Italian OW number, the expected SCs per year are approximately 2561 (279 MMs and 2282 NMSCs). INAIL recognised as occupational disease n. 246 cases (20\% MM vs $80 \%$ NMSCs) in the last 5 years, i.e. less than 50 cases per year.

Discussion and conclusions Our results show that, in Italy, the National Compensation Authority recognises less than the 2\% of the cases expected to occur in OW each year: 50 vs 2500. Main limitations of these data are that the incidence rates applied to OWs were not standardised, that the number of solar UV related SCs calculated is possibly under-estimated, considering that, e.g., not all OW groups were included, and that the data from cancer registries were quite outdated while the SCs incidence is increasing.

In conclusion, our data suggest a large under-estimation of occupational SCs in Italy, and that a better recognition of these diseases in OW is a relevant, and urgent, problem.

\section{GLIOMAS INCIDENCE IN ITALY}

${ }^{1}$ Alberto Modenese*, ${ }^{2}$ Leena Korpinen, ${ }^{1}$ Fabriziomaria Gobba. 'Department of Biomedical, Metabolic and Neural Sciences, University of Modena and Reggio Emilia, Modena, Italy; ${ }^{2}$ Clinical Physiology and Neurophysiology Unit, North Karelia Central Hospital and Honkalampi, Joensuu, Finland

\subsection{6/oemed-2018-ICOHabstracts.1230}

Introduction The International Agency for Research on Cancer has classified Radio Frequency in the Group 2B, 'possibly carcinogenic to humans', based on an association found between exposure related to mobile phones use and risk of gliomas and acoustic neuromas, even if this classification was discussed for some inconsistencies. Considering the large increase in mobile phone users from the ' $80 \mathrm{~s}$, an increase in incidence of these tumours should be expected. The aim of this work is to follow the incidence of gliomas in Italy.

Methods The national incidence of central nervous system (CNS) cancers and that of gliomas from 2006 was obtained from the Italian Cancer Registries

Result Considering CNS cancers, in the period 1996-2009 the yearly age-standardised incidence per 100000 has decreased from 10.8 to 9.8 in males, and from 8.0 to 7.0 in females. The incidence of gliomas, available for years 2006-2009 only, shows a slight increase from 5.7 to 6.3 in males, while is substantially stable (around 4) in females.

Discussion and conclusion Since the late '90 s, mobile phone use in Italy has largely increased: according to World Bank data, rose from 11.3 mobile phones per 100 Italians in 1996, to 107.7 in 2004 (exceeding the number of inhabitants), and 142.1 in 2015. If RF esposure related to mobile phones is associated with gliomas, considering a possible latency of 10 20 years an increase of the incidence of this tumours should be expected, at least from the second half of the 2000s

Considering all CNS tumours, during the period 19962009 data show a decreasing trend in Italy. For glioma, currently available standardised incidence rates, covering only the period 2006-2009, suggest a slight increase, but limited to males. Data available at this moment do not adequately support any firm conclusion on the trends of these tumours in Italy. 


\section{Reproductive Hazards}

\section{REPRODUCTIVE HAZARD IN THE WORKPLACE AND ENVIRONMENT}

Pau-Chung Chen*. Department of Public Health and Institute of Occupational Medicine and Industrial Hygiene, National Taiwan University College of Public Health, Taipei, Taiwan

\subsection{6/oemed-2018-ICOHabstracts. 1231}

\section{Aim of special session}

1. Provide new knowledge to prevent reproductive hazards.

2. Build mutual networks among safety and health experts

${ }^{1}$ Soile Jungewelter, ${ }^{2}$ Imane EL Amri, ${ }^{3,4}$ Andrea HiddingaSchipper, ${ }^{5}$ Ayman Fahim, ${ }^{6}$ Teus Brand, ${ }^{7}$ Ching-Chun Lin

${ }^{1}$ Finnish Institute of Occupational Health, Helsinki, Finland

${ }^{2}$ Faculty of Medicine and Pharmacy of Casablanca, Casablanca, Morocco

${ }^{3}$ National Institute for Insurance against Accidents at workDepartment of Occupational and Environmental Medicine, Epidemiology and Hygiene, Monteporzio Catone (RM), Italy

${ }^{4}$ Arbo Unie, Utrecht, The Netherlands

${ }^{5}$ Faculty Of Medicine, Suez Canal University, Ismailia, Egypt

${ }^{6}$ Netherlands Centre for Occupational Diseases, Coronel Institute of Occupational Health, Academic Medical Centre, Amsterdam, The Netherlands

${ }^{7}$ Institute of Occupational Medicine and Industrial Hygiene, National Taiwan University College of Public Health, Taipei, Taiwan

\section{$1727 a$ IS OCCUPATIONAL NOISE EXPOSURE DURING PREGNANCY RELATED TO LANGUAGE ACQUISITION OF THE CHILD?}

\footnotetext{
1,2Soile Jungewelter* ${ }^{*}, 2$ Helena Taskinen, ${ }^{3}$ Eira Jansson-Verkasalo, ${ }^{1,4}$ Marija-Liisa Lindbohm, ${ }^{1}$ Jouko Remes, ${ }^{5}$ Minna Huotilainen, ${ }^{1}$ Markku Sallmén. ${ }^{1}$ Finnish Institute of Occupational Health, Helsinki, Finland; ${ }^{2}$ University of Helsinki, Helsinki, Finland; ${ }^{3}$ University of Turku, Department of Psychology and Speech, Turku, Finland; ${ }^{4}$ University of Tampere, Tampere, Finland; ${ }^{5}$ University of Helsinki, Cognitive Brain Research Unit, Helsinki, Finland
}

\subsection{6/oemed-2018-ICOHabstracts. 1232}

Introduction We examined the impact of noise exposure during pregnancy on the child's language acquisition at the age of one and two years.

Methods We conducted a cohort study among women working in the food industry, as kindergarten teachers, musicians, dental nurses and pharmacists with a new born child. At the age of one year the analyses included 408 mother-child pairs and at the age of two years 288. The mother filled the baseline questionnaire before the child was 12 months old, and the language acquisition questionnaire when the child was 12 months (Infant-Toddler Checklist, ITC) and 24 months (MacArthur Communicative Development Inventories, MCDI) old. An occupational hygienist assessed the noise exposure in three categories: no exposure, low exposure, moderate/high exposure. We analysed the data using linear regression.
Results At the age of one there were no statistically significant differences among the adjusted mean language acquisition ITC-scores of the children in different exposure categories. The adjusted scores among boys were 30.1 (95\% CI: 28.3 to 31.8 ) for no exposure, $29.7(27.4-32.0)$ for low noise, and 29.3 (26.7-31.9) for moderate/high exposure. Among girls these were 33.7 (31.9-35.5), 33.8 (31.3-36.4), and 33.6 (31.3-36.0), respectively. No associations were found in analyses of social communication, speech production and language comprehension. Noise exposure was associated with lower scores among kindergarten teachers. At the age of two mean MCDI-scores did not differ significantly between the noise exposure groups. The adjusted mean scores for expressive vocabulary among girls were 295 (95\% CI: 254 to 336) for no exposure, 303 (243-362) for low exposure, and 269 (212-326) for moderate/high exposure. Among boys the scores were 200 (154-246), 178 (111-246), and 225 (153-298), respectively.

Discussion We found no clear association between noise exposure during pregnancy and language acquisition among oneyear- or two-year-old children.

\section{7b PREMATURE BIRTH AND WORKING CONDITIONS: A PROSPECTIVE STUDY ABOUT 68 PREGNANT WOMEN}

${ }^{1}$ I El Amri ${ }^{*},{ }^{2} \mathrm{~F}$ Benbrahim, ${ }^{1} \mathrm{~B}$ Benali, ${ }^{1} \mathrm{~A}$ El Kholti. ${ }^{1}$ Faculty of Medicine and Pharmacy, Casablanca, Morocco; ${ }^{2}$ Faculty of Medicine and pharmacy, Rabat, Morocco

\subsection{6/oemed-2018-ICOHabstracts.1233}

Introduction The question about mediation between work and pregnancy is a real problem in industrialised countries, but in a country like ours, the issue is far away from being taken as a priority. Thus, we've chosen to conduct this prospective study about premature birth and working conditions trying to find a correlation between the two, while removing medical and obstetrical risk factors.

Methods The study was conducted in between January and December 2014. We've included women who had a job 6 months or more before the conception. To evaluate working conditions we've used the fatigue score developed by Mamelle. To evaluate the impact of working conditions on premature birth, we've monitored cervical length changes.

Results At first, we've gathered a selection of 68 pregnant women debuting their follow up between 11 and 13 weeks of amenorrhea plus 5 days. An acceleration of the modification of the uterine cervix was noted within the group of women having a «Mamelle» score of 3 .

Discussion The first epidemiological study bringing up the part of tiring working conditions in premature birth was conducted by Mamelle in France. After that, more authors, such as Mac Donald, got interested by the subject and implicated long working hours, rotating shifts and carrying heavy loads in the occurrence of premature birth. We took $18 \mathrm{~mm}$ as a limiting value for the length of the cervix under which intervention measures should be taken.

Conclusion Risk assessment of working conditions is the only deciding factor when it comes to the pursuit of work while being pregnant. The decision needs to be taken by both the attending and occupational physicians. 